\title{
Observation of microwave induced resistance and photovoltage oscillations in $\mathrm{MgZnO} / \mathrm{ZnO}$ heterostructures
}

\author{
D. F. Kärcher, ${ }^{1}$ A. V. Shchepetilnikov, ${ }^{2}$ Yu. A. Nefyodov, ${ }^{2}$ J. Falson, ${ }^{1,3}$ I. A. Dmitriev, ${ }^{1,4}$ Y. Kozuka, ${ }^{3}$ D. Maryenko, ${ }^{5}$ \\ A. Tsukazaki, ${ }^{6,7}$ S. I. Dorozhkin, ${ }^{2}$ I. V. Kukushkin, ${ }^{2}$ M. Kawasaki, ${ }^{3,5}$ and J. H. Smet ${ }^{1, *}$ \\ ${ }^{1}$ Max Planck Institute for Solid State Research, D-70569 Stuttgart, Germany \\ ${ }^{2}$ Institute of Solid State Physics RAS, 142432 Chernogolovka, Moscow District, Russia \\ ${ }^{3}$ Department of Applied Physics and Quantum-Phase Electronics Center (QPEC), The University of Tokyo, Tokyo 113-8656, Japan \\ ${ }^{4}$ A. F. Ioffe Physico-Technical Institute, 194021 St. Petersburg, Russia \\ ${ }^{5}$ RIKEN Center for Emergent Matter Science (CEMS), Wako 351-0198, Japan \\ ${ }^{6}$ Institute for Materials Research, Tohoku University, Sendai 980-8577, Japan \\ ${ }^{7}$ PRESTO, Japan Science and Technology Agency (JST), Tokyo 102-0075, Japan
}

(Received 29 September 2015; published 15 January 2016)

\begin{abstract}
Microwave induced resistance and photovoltage oscillations were investigated in $\mathrm{Mg}_{x} \mathrm{Zn}_{1-x} \mathrm{O} / \mathrm{ZnO}$ heterostructures. The physics of these oscillations is controlled significantly by scattering mechanisms, and therefore these experiments were motivated by the recently achieved high quality levels in this material and the apparent dominance of large angle, short-range scattering, which is distinct from the prevailing small angle scattering in state-of-the-art GaAs structures. Within the studied frequency range of $35-120 \mathrm{GHz}$, up to four oscillations were resolved at $1.4 \mathrm{~K}$ temperature, but only in high density samples. This allowed us to extract the value of the effective electron mass $m^{*}=(0.35 \pm 0.01) m_{0}$, which is enhanced over the bare band mass, and estimate a local quantum scattering time of about 5 ps.
\end{abstract}

DOI: 10.1103/PhysRevB.93.041410

The irradiation of two-dimensional electron systems (2DES) with microwaves elicits a rich variety of phenomena [1]. Microwave induced resistance oscillations (MIROs) [2-6] are the most celebrated member in the family of nonequilibrium magneto-oscillations. Phenomenologically, the oscillatory change in the dissipative resistance $R_{x x}$ induced by the incident microwave irradiation $\Delta R_{x x}^{\text {osc }}$ is usually well described by a damped sine wave

$$
\Delta R_{x x}^{\mathrm{osc}}=-\epsilon A_{\epsilon} \sin (2 \pi \epsilon) \exp (-\alpha \epsilon) .
$$

Here, $\epsilon=\omega / \omega_{c}$ is the ratio of the cyclic microwave frequency $\omega=2 \pi f$ and the cyclotron frequency $\omega_{c}=e B / m^{*}$. Extrema are then located at quarter values of $\epsilon=j \pm 1 / 4$, where $j=1,2, \ldots$. The exponential describes the damping of the oscillations and $\alpha$ is the damping factor ( $-e$ being the electron charge, $B$ the magnetic field perpendicular to the 2DES, and $m^{*}$ the effective mass). According to the most frequently used models to account for MIROs referred to as the displacement [7-11] and inelastic [12-14] mechanisms, the damping coefficient $\alpha=2 \pi / \omega \tau_{q}$ is proportional to the quantum scattering rate $\tau_{q}^{-1}$ (see Ref. [15]). The prefactor $A_{\epsilon}$ depends on the incident microwave power and becomes $B$ independent for $\epsilon \gg 1$ in both theory and experiment. The Hall resistance $R_{x y}=B / e n$, where $n$ is the electron areal density, remains classical and unaffected by the radiation in the range of $B$ where MIROs have been observed so far [16-19], apart from changes that are in absolute terms comparable in size to the changes in the longitudinal resistivity. The microwave radiation does not only induce resistance oscillations periodic in the inverse of the magnetic field, but also generates photovoltage or photocurrent oscillations [20,21] with the same periodicity in samples with internal contacts due to

\footnotetext{
*j.smet@fkf.mpg.de
}

intrinsic internal electric fields associated with sample disorder and density inhomogeneities, for instance, near contacts [22]. These photovoltage or photocurrent oscillations are closely related to MIROs. In particular, in the regime of weak MIROs, the photovoltage oscillations are proportional to $\Delta R_{x x}^{\text {osc }}$ (see Refs. [22,23]).

The initial stage of research indicated that MIROs become more pronounced in samples with higher mobility $\mu=e \tau / m^{*}$ (i.e., lower momentum relaxation rate $\tau^{-1}$ ). Indeed, while first observations [2,3] in $\mathrm{Al}_{x} \mathrm{Ga}_{1-x} \mathrm{As} / \mathrm{GaAs}$ heterostructures with $\mu \sim 3 \times 10^{6} \mathrm{~cm}^{2} / \mathrm{V}$ s reported $\Delta R_{x x}^{\text {osc }} / R_{x x} \sim 0.1$, in later measurements on samples with $\mu=15-25 \times 10^{6} \mathrm{~cm}^{2} / \mathrm{V} \mathrm{s}$, zero-resistance states (ZRS) with $R_{x x} \rightarrow 0$ in low-order minima of MIROs were discovered [4-6]. These ZRS are attributed [24] to domain instability which emerges when the amplitude of MIROs exceeds the dark resistivity and turns the overall resistivity of the uniform system negative $[9,10,12,14]$. The domain nature of ZRS is supported by recent local time-resolved measurements [25,26].

More systematic studies have shown, however, that, similar to some other fragile transport phenomena such as even denominator fractional quantum Hall physics at filling 5/2, there is no apparent correlation between MIROs and the sample mobility [27,28]. Theory has also confirmed that additional disorder characteristics are relevant for the magnitude of MIROs. Neither the transport scattering time $(\tau$, or alternatively, mobility) nor the quantum lifetime $\left(\tau_{q}\right)$, which determines the width of the disorder-broadened Landau levels, are sufficient. For instance [11], within the model of weak Gaussian disorder with a scattering angle $\theta$ dependent scattering rate $\tau_{\theta}^{-1}$, the displacement contribution to MIROs is proportional to $\tau_{\star}^{-1}=2\left\langle\tau_{\theta}^{-1}(1-\cos \theta)^{2}\right\rangle$. Here, \langle\rangle denotes the angular average. This dependence is distinct from the quantum scattering rate $\tau_{q}^{-1}=\left\langle\tau_{\theta}^{-1}\right\rangle$ and the transport scattering rate $\tau^{-1}=\left\langle\tau_{\theta}^{-1}(1-\cos \theta)\right\rangle$. As a result, the MIRO amplitude 
may vary by orders of magnitude when modifying the relative strength of the long- and short-range components of disorder even though both the transport and quantum scattering rates are kept fixed [15]. The above argument shows that MIROs serve as a probe of properties of the 2DES that are distinct from those accessible in standard low- $B$ transport measurements. The detection of MIROs in materials with disorder possessing different correlations may be beneficial to gain a deeper understanding of the microscopic mechanisms of MIROs and nonequilibrium transport in general.

Here, we report the observation of MIROs and the closely related photovoltage oscillations in high-mobility oxide $\mathrm{Mg}_{x} \mathrm{Zn}_{1-x} \mathrm{O} / \mathrm{ZnO}$ heterojunctions which, due to recent technological advances, approach the quality of state-ofthe-art AlGaAs/GaAs heterostructures, as evidenced by the observation of fragile fractional quantum Hall states [29,30]. The different doping mechanism as well as significantly larger band effective mass $\left(m_{b}^{*}=0.3 m_{0}\right.$, where $m_{0}$ is the free electron mass) sets this system apart from $\mathrm{AlGaAs} / \mathrm{GaAs}$ [1] and $p$-type SiGe/Ge [31], the two semiconductor based systems in which MIROs have been observed so far (very recently, MIROs were also observed in a non-degenerate electron system above the surface of liquid helium [32]). The unique character of the $\mathrm{MgZnO} / \mathrm{ZnO}$ system has also been highlighted in a recent study of the transport time and quantum lifetime in this material system, which indicated that disorder and scattering mechanisms are distinct from those prevalently operating in $\mathrm{AlGaAs} / \mathrm{GaAs}$ [33]. A short-range disorder potential causing larger angle scattering plays a much more significant role in the $\mathrm{MgZnO} / \mathrm{ZnO}$ system. This is not surprising since in state-of-the-art GaAs samples the random distribution of the remote donors are the important source of scattering, whereas in $\mathrm{MgZnO} / \mathrm{ZnO}$ based systems the difference in surface polarization between the $\mathrm{MgZnO}$ alloy and $\mathrm{ZnO}$ generates the two-dimensional (2D) system, and the heterointerface itself likely hosts most of the disorder due to the random distribution of the $\mathrm{Mg}$ atoms.

The $\mathrm{Mg}_{x} \mathrm{Zn}_{1-x} \mathrm{O} / \mathrm{ZnO}$ heterostructures were grown using liquid ozone based molecular beam epitaxy [34]. The MIROs were observed on a number of samples in the Hall bar, Corbino, and van der Pauw geometries. Dimensions were $2 \times 2.5 \mathrm{~mm}$ with $1 \mathrm{~mm}$ between voltage probes for the Hall bar, 0.4 and $1.0 \mathrm{~mm}$ inner and outer diameters for the Corbino device, and $3 \mathrm{~mm}^{2}$ for the van der Pauw chip. Here, we focus on two samples with the $\mathrm{Mg}$ content $x \simeq(0.04,0.055)$ corresponding to the electron density $n=(6.5,8.5) \times 10^{11} \mathrm{~cm}^{-2}$ and mobility $\mu=(1,0.5) \times 10^{5} \mathrm{~cm}^{2} / \mathrm{V}$ s. For all sample geometries, ohmic contacts were made by evaporated Ti/Au or soldered indium. The experiments were performed at temperatures $T \approx 1.4 \mathrm{~K}$ in unipolar magnetic fields up to $10 \mathrm{~T}$. Samples were irradiated with microwave radiation ( $f=35-120 \mathrm{GHz}$ ) guided to the chip via an oversized waveguide. A $K_{u}$-band generator with frequency multipliers, backward wave oscillators (BWOs), and a fixed-frequency impact ionization avalanche transit-time (IMPATT) diode were used as millimeter wave sources. While the multipliers' output power is limited to $4 \mathrm{~mW}$, the IMPATT diode provides much higher power, roughly $100 \mathrm{~mW}$, at a fixed frequency of $97 \mathrm{GHz}$. In the remainder of this Rapid Communication, power values refer to the output power of the source. A determination of the power incident on the sample is challenging as the waveguide is operated in oversized mode and the microwave field distribution may vary erratically with frequency due to standing waves. Hence, only data recorded at identical frequencies but with different relative power of the microwave radiation can be compared when addressing amplitude effects. The longitudinal resistance of the sample was acquired with a low frequency lock-in technique and an imposed alternating current of $1 \mu \mathrm{A}$. In cases where increased sensitivity is necessary, a double modulation technique was implemented. The frequency of the imposed current was then increased to $2 \mathrm{kHz}$. The microwave amplitude was modulated at a frequency $f_{\text {mod }}$ two orders of magnitude lower than the ac-current frequency. A first lock-in amplifier was tuned to the frequency of the alternating current to detect the resistance. Its time constant was set short enough to ensure that its analog output still contained the modulation by the microwave radiation at frequency $f_{\text {mod }}$. This alternating output signal served as the input to a second lock-in amplifier tuned to $f_{\text {mod }}$ in order to measure the change in the resistance caused by the microwave irradiation.

Figure 1(a) compares $R_{x x}$ of a Hall bar device fabricated on the heterostructure with $n=6.5 \times 10^{11} \mathrm{~cm}^{-2}$ when the microwave radiation of the IMPATT diode $(f=97 \mathrm{GHz})$ is incident on the sample (solid line) and absent (dotted line). The magnetic field was swept while the frequency and power of the radiation were kept constant. In addition to an overall increase in resistance due to heating of the sample from the microwaves, a robust oscillatory modulation is apparent. In Fig. 1(b), the difference $\Delta R_{x x}$ between the two traces is shown. A number of oscillatory features are resolved, with the most robust minimum at $B \approx 1 \mathrm{~T}$ corresponding to $\epsilon=\epsilon_{1-}=5 / 4$ (the index refers to the order of the extremum and the sign to a minimum or maximum). Fitting the data presented in Fig. 1(b) using Eq. (1) with $A_{\epsilon}=$ const (dotted line) yields an estimate of the quantum time $\tau_{q}=5 \mathrm{ps}$. This value is in good agreement with that obtained through an analysis of Shubnikov-de Haas oscillations (SdHOs) [33], although SdHOs frequently yield shorter values. The reason for this discrepancy is that the MIRO phase $2 \pi \epsilon$ is insensitive to the electron density, while the SdHO phase is proportional to it. As a result, long-range density variations do not affect MIROs while strongly reducing the amplitude of SdHOs [1]. In other words, the $\tau_{q}$ deduced from SdHOs includes an inhomogeneous broadening of Landau levels, while MIROs measure the local $\tau_{q}$.

Data at other frequencies were obtained with full-band multipliers. Because these have two orders of magnitude lower power, the higher sensitivity double modulation technique was exploited for the detection of the oscillations. Figure 1(c) shows an example of MIROs measured with this technique for low power microwaves at the same frequency as the IMPATT diode $(97 \mathrm{GHz})$. The measurement was performed in Corbino geometry with the data representing the microwave induced variation $\left(1 / G_{\text {dark }}-1 / G_{\mathrm{mw}}\right) \times 10^{3} G_{\text {dark }}$, with $G_{\mathrm{mw}}\left(G_{\text {dark }}\right)$ being the Corbino ring conductance in the presence (absence) of the microwave illumination. The period and phase of the oscillations coincide with those for $\Delta R_{x x}$ measured on the Hall bar device in Fig. 1(b). This phase is examined in more detail for the Hall bar data of Fig. 1(b) in the inset by plotting those extrema not cluttered by remnants of the Shubnikov-de Haas 


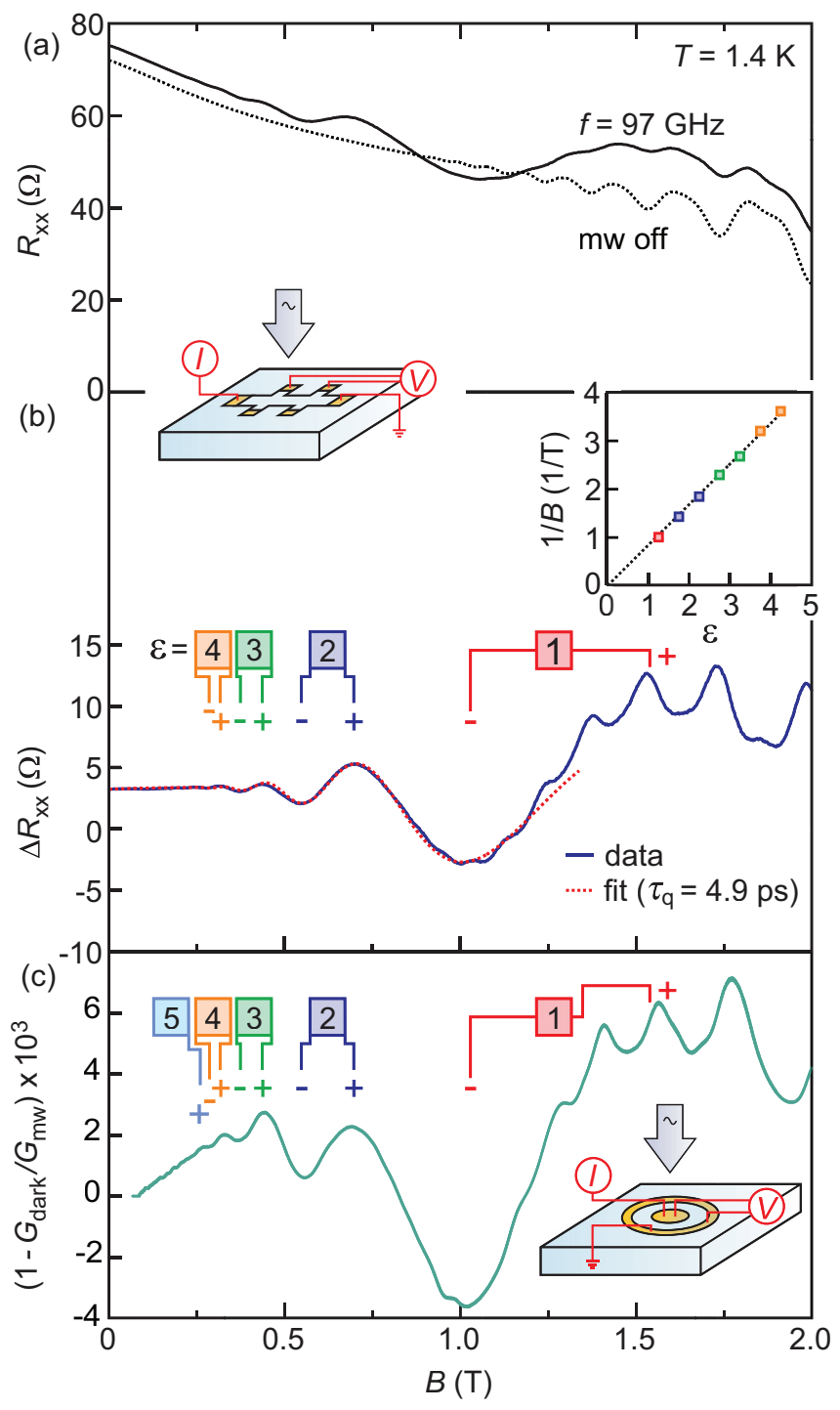

FIG. 1. The magnetic field dependences of (a) the Hall bar longitudinal resistance with (solid) and without (dotted) radiation from the IMPATT diode. (b) $\Delta R_{x x}$ of the two traces shown in (a). The inset displays the reciprocal values of the magnetic field corresponding to the MIRO extrema vs $\epsilon$. (c) The microwave photoresponse $1 / G_{\mathrm{mw}}-1 / G_{\text {dark }}$ in the inverse Corbino conductance, i.e., the two-point Corbino resistance, measured by the double lock-in technique is shown multiplied by $\left(-G_{\text {dark }}\right)$ to compensate the strong monotonic dependence on the magnetic field and to allow a straightforward comparison of oscillating behavior with that in $\Delta R_{\mathrm{xx}}$. For all traces, $f=97 \mathrm{GHz}$.

oscillations. Assuming, in accordance with Eq. (1), that the extrema occur at $\epsilon=j \pm 1 / 4$ with $j$ an integer, experimental points are well fitted by the same straight line passing through the origin. Hence, the phase and period of MIROs are in line with previous observations [35].

In Fig. 2 we plot the oscillation extrema as a function of the magnetic field for the full range of the microwave frequencies explored. Their positions were determined by fitting a parabola near the local extrema marked in Fig. 1(c) (excluding the $\epsilon_{1+}$ maximum strongly corrugated by the SdHOs). The solid lines in Fig. 2 are obtained by linear least-square fits to each set

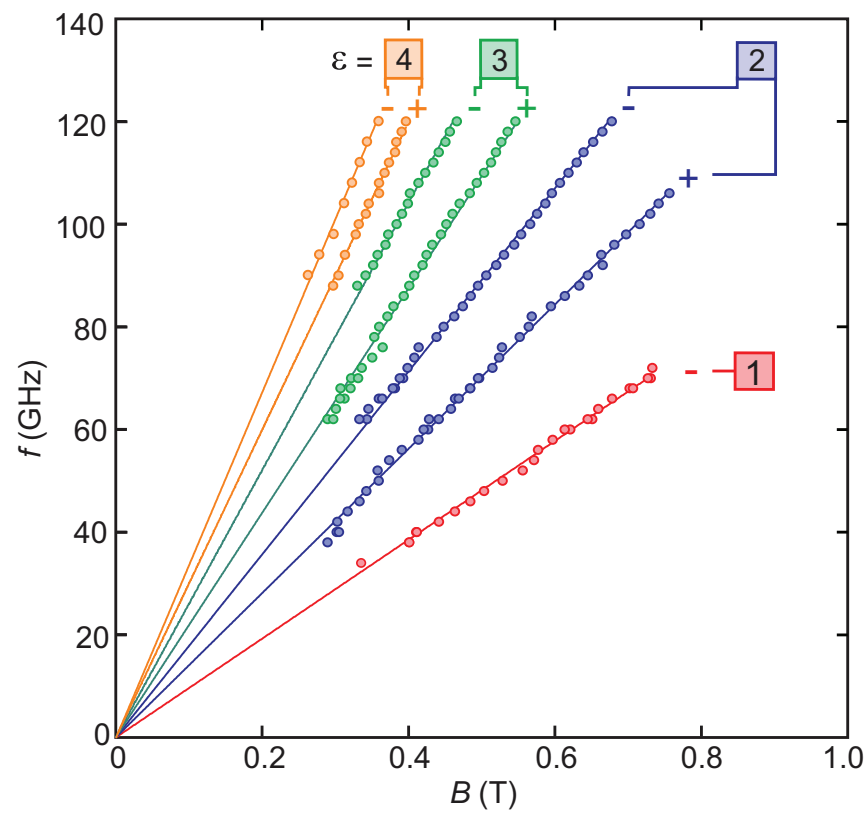

FIG. 2. The microwave irradiation frequency against the magnetic field for the different extrema at $\epsilon=j \pm 1 / 4$. Straight lines are fits to the data with the effective mass as a fitting parameter (see text for details).

of extrema under the assumption that these occur when $\epsilon=$ $j \pm 1 / 4$ with the carrier effective mass as the fitting parameter. The good quality fit obtained allows a precise measurement of the mass, which is found to be $m^{*}=(0.35 \pm 0.01) m_{0}$. The mass value extracted from MIROs is close to the effective mass obtained from magnetoplasmon resonances [36], which is similarly larger than the band mass $\left(m_{b}^{*}=0.3 m_{0}\right)$.

We now turn our attention to photovoltage measurements, which are known to possess a phase and period similar to MIROs but require an alternative geometry containing internal contacts to the 2DES [20-22]. Photovoltaic effects are understood in terms of a modified relation between conductivity and diffusion under the microwave illumination [22,23]. As a result, the steady-state distribution of the electron density in an inhomogeneous 2DEG (for instance, near internal metallic contacts) no longer corresponds to a constant electrochemical potential, which results in a photovoltage detected via local probes [37]. These experiments were carried out on the sample with $n=8.5 \times 10^{11} \mathrm{~cm}^{-2}$. It is schematically illustrated in the inset to Fig. 3(b). The voltage is tapped off between an internal and external contact while no current is imposed. The microwave radiation is chopped so the photovoltage can be recorded with a single lock-in detection technique. In Fig. 3(a) we present an example of such photovoltage oscillations. An analysis similar to that in Fig. 2 (not shown) yielded $m^{*}=(0.330 \pm 0.005) m_{0}$. The traces in Fig. 3(a) were recorded at different levels of the output power of the microwave source. The actual power incident on the sample may differ substantially from the indicated values, so only the power ratios are relevant. To analyze these photovoltage oscillations, a fourth-order polynomial function was fitted to each data trace from $B=0$ through points where $\epsilon$ takes on integer values using the experimentally determined value of 

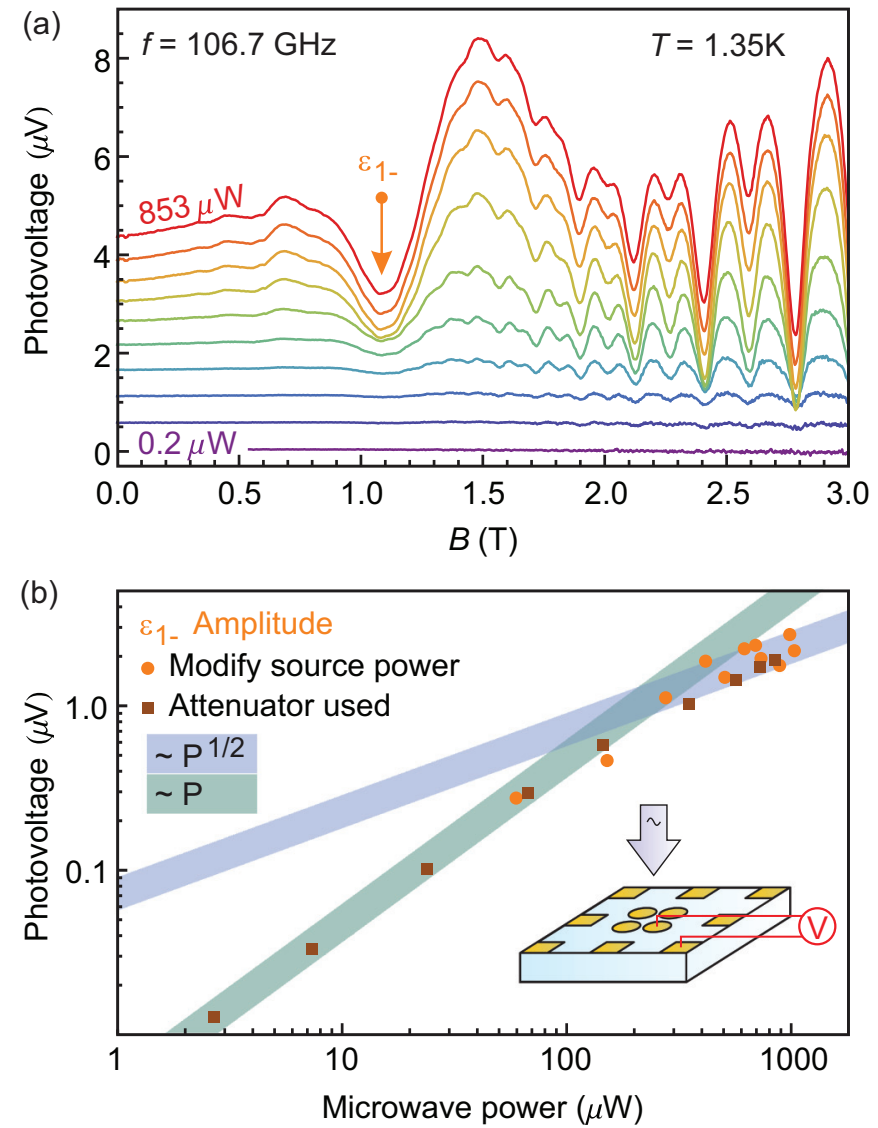

FIG. 3. (a) Photovoltage measurements under microwave radiation $(f=106.7 \mathrm{GHz})$ between internal and external contacts for different microwave powers. Each curve is offset by $0.5 \mu \mathrm{V}$ for clarity. (b) Power dependence of the amplitude of the $\epsilon_{1-}$ minimum. The power value is that measured at the wave source. Two distinct regimes are resolved with a crossover at $P=200 \mu \mathrm{W}$. At low power the dependence is linear, and at higher power the data points follow a sublinear dependence. The inset shows the sample measurement geometry.

$m^{*}=0.33 m_{0}$. The amplitude relative to the nodes is then determined. Figure 3(b) shows the amplitude of the $\epsilon_{1-}$ minimum. It has been evaluated over a power range covering nearly three orders of magnitude. The amplitude exhibits a transition from the linear $A_{\epsilon} \propto P$ to a sublinear dependence near $P=200 \mu \mathrm{W}$. While at the present stage it is unclear which mechanism is responsible for the observed saturation, possible mechanisms include a feedback effect in the inelastic mechanism [14], multiphoton processes in the displacement mechanism [11,38,39], and a heating induced width change of the Landau levels via electron-electron interaction effects $[15,40]$.

We note that MIROs and photovoltage oscillations were also explored in $\mathrm{MgZnO} / \mathrm{ZnO}$ samples with lower densities down to $n \leqslant 4 \times 10^{11} \mathrm{~cm}^{-2}$. Despite the order of magnitude
TABLE I. Intermaterial parameter comparison of $m^{*} / m_{0}$, density, mobility, and $\tau_{q}$ for a number of reports on MIRO in the literature (Refs. [28,31,41] and this work).

\begin{tabular}{llccc}
\hline \hline & $m^{*} / m_{0}$ & $\begin{array}{c}\text { Density } \\
\left(10^{11} \mathrm{~cm}^{-2}\right)\end{array}$ & $\begin{array}{c}\text { Mobility } \\
\left(\mathrm{cm}^{2} / \mathrm{V} \mathrm{s}\right)\end{array}$ & $\begin{array}{c}\tau_{q} \\
(\mathrm{ps})\end{array}$ \\
\hline AlGaAs/GaAs, Ref. [41] & 0.067 & 1.9 & $10 \times 10^{6}$ & 15 \\
Reference [28] & 0.067 & 8.5 & $5.6 \times 10^{5}$ & \\
SiGe/Ge, Ref. [31] & 0.091 & 2.8 & $4 \times 10^{5}$ & 4 \\
MgZnO/ZnO & 0.35 & $>6$ & $5 \times 10^{4}$ & 5 \\
\hline \hline
\end{tabular}

higher mobility and quantum lifetime these samples exhibited [33], this search was in vain. Instead, a resistance peak attributed to the hybrid dimensional magnetoplasmon cyclotron resonance mode was exclusively observed. It is a striking confirmation that neither the transport scattering rate nor the quantum scattering rate capture the essential criteria which the sample disorder has to fulfill to make MIROs observable. Table I summarizes the key parameters of samples in the different semiconductor material systems in which MIROs have been observed so far. The absence of MIROs in $\mathrm{ZnO}$ in lower density samples is also in line with observations on GaAs heterostructures, where even modest mobility samples may show the effect provided the charge carrier density is sufficiently high, as shown in the second row of Table I. Both the momentum relaxation time and the electron density of the $\mathrm{ZnO}$ samples which reveal MIROs are

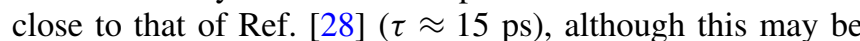
coincidental. Equally remarkable is the apparent insensitivity of MIROs to the cyclotron energy and level splitting. The effective mass of the $\mathrm{ZnO}$ conduction band is up to five times larger in comparison with GaAs and $p$-Ge in which MIROs have been observed previously and yet the frequencies explored here are identical. While the basic phenomenology is accurately described by existing theoretical models, the apparent relevance of high density for samples with moderate mobility, together with the insensitivity of this effect to the handedness of circular polarization [42], remains poorly understood and controversial $[1,43]$. With its very different material parameters, $\mathrm{MgZnO} / \mathrm{ZnO}$ heterostructures now offer an additional platform to explore these unresolved questions.

We gratefully acknowledge financial support from the Japan Society for the Promotion of Science through a Grant-in-Aid for Scientific Research(S) No. 24226002 and for Young Scientists (A) No. 23686008 as well as from the "Funding Program for World-Leading Innovative R\&D on Science and Technology (FIRST Program)" initiated by the Council for Science and Technology Policy (CSTP). A.V.S., Y.A.N., S.I.D., and I.V.K. thank the Russian Science Foundation (Grant No. 14-12-00599) for support in developing microwave measurements on $\mathrm{ZnO}$ heterostructures.
[1] I. A. Dmitriev, A. D. Mirlin, D. G. Polyakov, and M. A. Zudov, Rev. Mod. Phys. 84, 1709 (2012).
[2] M. A. Zudov, R. R. Du, J. A. Simmons, and J. L. Reno, Phys. Rev. B 64, 201311(R) (2001). 
[3] P. D. Ye, L. W. Engel, D. C. Tsui, J. A. Simmons, J. R. Wendt, G. A. Vawter, and J. L. Reno, Appl. Phys. Lett. 79, 2193 (2001).

[4] R. G. Mani, J. H. Smet, K. von Klitzing, V. Narayanamurti, W. B. Johnson, and V. Umansky, Nature (London) 420, 646 (2002).

[5] M. A. Zudov, R. R. Du, L. N. Pfeiffer, and K. W. West, Phys. Rev. Lett. 90, 046807 (2003).

[6] C. L. Yang, M. A. Zudov, T. A. Knuuttila, R. R. Du, L. N. Pfeiffer, and K. W. West, Phys. Rev. Lett. 91, 096803 (2003).

[7] V. I. Ryzhii, Sov. Phys. Solid State 11, 2078 (1970).

[8] V. I. Ryzhii, R. A. Suris, and B. S. Shchamkhalova, Sov. Phys. Semicond. 20, 1299 (1986).

[9] A. C. Durst, S. Sachdev, N. Read, and S. M. Girvin, Phys. Rev. Lett. 91, 086803 (2003).

[10] M. G. Vavilov and I. L. Aleiner, Phys. Rev. B 69, 035303 (2004).

[11] M. Khodas and M. G. Vavilov, Phys. Rev. B 78, 245319 (2008).

[12] S. I. Dorozhkin, JETP Lett. 77, 577 (2003).

[13] I. A. Dmitriev, A. D. Mirlin, and D. G. Polyakov, Phys. Rev. Lett. 91, 226802 (2003).

[14] I. A. Dmitriev, M. G. Vavilov, I. L. Aleiner, A. D. Mirlin, and D. G. Polyakov, Phys. Rev. B 71, 115316 (2005).

[15] I. A. Dmitriev, M. Khodas, A. D. Mirlin, D. G. Polyakov, and M. G. Vavilov, Phys. Rev. B 80, 165327 (2009).

[16] R. G. Mani, V. Narayanamurti, K. von Klitzing, J. H. Smet, W. B. Johnson, and V. Umansky, Phys. Rev. B 69, 161306(R) (2004).

[17] S. A. Studenikin, M. Potemski, P. T. Coleridge, A. S. Sachrajda, and Z. R. Wasilewski, Solid State Commun. 129, 341 (2004).

[18] S. Wiedmann, G. M. Gusev, O. E. Raichev, S. Krämer, A. K. Bakarov, and J. C. Portal, Phys. Rev. B 83, 195317 (2011).

[19] I. A. Dmitriev, A. D. Mirlin, and D. G. Polyakov, Phys. Rev. B 75, 245320 (2007).

[20] R. L. Willett, L. N. Pfeiffer, and K. W. West, Phys. Rev. Lett. 93, 026804 (2004).

[21] A. A. Bykov, JETP Lett. 87, 233 (2008).

[22] S. I. Dorozhkin, I. V. Pechenezhskiy, L. N. Pfeiffer, K. W. West, V. Umansky, K. von Klitzing, and J. H. Smet, Phys. Rev. Lett. 102, 036602 (2009).

[23] I. A. Dmitriev, S. I. Dorozhkin, and A. D. Mirlin, Phys. Rev. B 80, 125418 (2009).

[24] A. V. Andreev, I. L. Aleiner, and A. J. Millis, Phys. Rev. Lett. 91, 056803 (2003).
[25] S. I. Dorozhkin, L. Pfeiffer, K. West, K. von Klitzing, and J. H. Smet, Nat. Phys. 7, 336 (2011).

[26] S. I. Dorozhkin, V. Umansky, L. N. Pfeiffer, K. W. West, K. Baldwin, K. von Klitzing, and J. H. Smet, Phys. Rev. Lett. 114, 176808 (2015).

[27] J. Nübler, Ph.D. thesis, Max Planck Institute for Solid State Research, Stuttgart, 2011.

[28] A. A. Bykov, A. K. Bakarov, D. R. Islamov, and A. I. Toropov, JETP Lett. 84, 391 (2006).

[29] A. Tsukazaki, S. Akasaka, K. Nakahara, Y. Ohno, H. Ohno, D. Maryenko, A. Ohtomo, and M. Kawasaki, Nat. Mater. 9, 889 (2010).

[30] J. Falson, D. Maryenko, B. Friess, D. Zhang, Y. Kozuka, A. Tsukazaki, J. H. Smet, and M. Kawasaki, Nat. Phys. 11, 347 (2015).

[31] M. A. Zudov, O. A. Mironov, Q. A. Ebner, P. D. Martin, Q. Shi, and D. R. Leadley, Phys. Rev. B 89, 125401 (2014).

[32] R. Yamashiro, L. V. Abdurakhimov, A. O. Badrutdinov, Yu. P. Monarkha, and D. Konstantinov, Phys. Rev. Lett. 115, 256802 (2015).

[33] J. Falson, Y. Kozuka, J. H. Smet, T. Arima, A. Tsukazaki, and M. Kawasaki, Appl. Phys. Lett. 107, 082102 (2015).

[34] J. Falson, D. Maryenko, Y. Kozuka, A. Tsukazaki, and M. Kawasaki, Appl. Phys. Express 4, 091101 (2011).

[35] R. G. Mani, J. H. Smet, K. von Klitzing, V. Narayanamurti, W. B. Johnson, and V. Umansky, Phys. Rev. Lett. 92, 146801 (2004).

[36] V. E. Kozlov, A. B. Van'kov, S. I. Gubarev et al., Phys. Rev. B 91, 085304 (2015).

[37] S. I. Dorozhkin, I. A. Dmitriev, and A. D. Mirlin, Phys. Rev. B 84, 125448 (2011).

[38] M. Khodas, H. S. Chiang, A. T. Hatke, M. A. Zudov, M. G. Vavilov, L. N. Pfeiffer, and K. W. West, Phys. Rev. Lett. 104, 206801 (2010)

[39] A. T. Hatke, M. Khodas, M. A. Zudov, L. N. Pfeiffer, and K. W. West, Phys. Rev. B 84, 241302(R) (2011).

[40] V. Ryzhii, A. Chaplik, and R. Suris, JETP Lett. 80, 363 (2004).

[41] S. A. Studenikin, M. Potemski, A. Sachrajda, M. Hilke, L. N. Pfeiffer, and K. W. West, Phys. Rev. B 71, 245313 (2005).

[42] J. H. Smet, B. Gorshunov, C. Jiang, L. Pfeiffer, K. West, V. Umansky, M. Dressel, R. Meisels, F. Kuchar, and K. von Klitzing, Phys. Rev. Lett. 95, 116804 (2005).

[43] S. A. Mikhailov, Phys. Rev. B 83, 155303 (2011). 